\title{
Long non-coding RNA LSINCT5 inactivates Wnt/ $\beta$-catenin pathway to regulate MCF-7 cell proliferation and motility through targeting the miR-30a
}

\author{
Guizhi Zhang', Wenbo Song ${ }^{2}$ \\ ${ }^{1}$ Department of Radiology, The Eighth Affiliated Hospital Sun Yat-sen University, Shenzhen, China; ${ }^{2}$ Department of Oncology, Jiangdu People's \\ Hospital Affiliated to Medical College of Yangzhou University, Yangzhou, China \\ Contributions: (I) Conception and design: All authors; (II) Administrative support: W Song; (III) Provision of study materials or patients: G Zhang; (IV) \\ Collection and assembly of data: G Zhang; (V) Data analysis and interpretation: G Zhang; (VI) Manuscript writing: All authors; (VII) Final approval \\ of manuscript: All authors. \\ Correspondence to: Wenbo Song. Department of Oncology, Jiangdu people's Hospital Affiliated to Medical College of Yangzhou University, No. 9 \\ Dongfanghong Road, Yangzhou, China. Email: zhangguizhi1234@163.com.
}

Background: Breast cancer (BC) is the most common malignant tumor among women. Earlier studies showed that long stress-induced non-coding transcript 5 (LSINCT5) was implicated in BC. However, the potential mechanisms of LSINCT5 in BC is still elusive.

Methods: Relative expression of LSINCT5 in BC tissues and cells were quantified by quantitative realtime reverse transcription PCR (qRT-PCR). shRNA was employed to specifically knockdown endogenous LSINCT5 in BC cells. Cell growth and invasion activity of BC cells was assessed by colony formation and transwell migration assay, respectively. The association between LSINCT5 and miR-30a was conducted by luciferase reporter assay. Subcutaneous injection of sh-LSINCT5 transfected MCF-7 cells into the ventral regions of mice to form tumors. Mice were divided into three groups $(\mathrm{n}=10)$ : control group, sh-NC group, sh-LSINCT5 group (sh-NC or sh-LSINCT5 transfected MCF-7 cells injected into mice). Tumor weight was checked after 30 days post-injection.

Results: LSINCT5 was significantly up-regulated in BC tissues and cells. LSINCT5 knockdown suppressed proliferation, invasion, and epithelial-mesenchymal transition (EMT) in vitro and in vivo. LSINCT5 acted as a sponge molecule and targeted miR-30a in BC cells. Further mechanistic study exhibited that overexpression of LSINCT5 promoted the expression of $\mathrm{Wnt} / \beta$-catenin-related proteins $(\beta$-catenin, TCF4, and c-Myc). In vivo, xenograft nude mice experiment indicated sh-LSINCT5 inhibited tumor growth and motility by targeting miR-30a through modulating $\mathrm{Wnt} / \beta$-catenin pathway.

Conclusions: The present results uncovered that LSINCT5 knockdown suppressed BC growth and metastasis via the miR-30a/Wnt/ $\beta$-catenin axis, and it served as a potential therapeutic target for early diagnosis and treatment of $\mathrm{BC}$ patients..

Keywords: Breast cancer (BC); long non-coding RNAs; microRNAs; proliferation; motility; Wnt/ $\beta$-catenin pathway

Submitted Jul 06, 2020. Accepted for publication Dec 13, 2020.

doi: $10.21037 / \mathrm{atm}-20-7253$

View this article at: http://dx.doi.org/10.21037/atm-20-7253

(C) Annals of Translational Medicine. All rights reserved. 


\section{Introduction}

As the most common malignant tumor among women, breast cancer (BC) displays a rapidly increasing morbidity and incidence in China (1). The high morbidity and incidence were attributed due to invasiveness, poor prognosis, and resistance against current therapies (2). Therefore, traditional therapies, including surgery, hormonotherapy, radiation therapy, and chemotherapy, were not efficacious in the treatment of advanced BC (3). It is still imperative to develop efficient therapies for the treatment of BC. The critical point to discover is how $\mathrm{BC}$ initiates, progresses, invades, and recurs. Thus, the underlying molecular mechanisms of $\mathrm{BC}$ still need to be addressed urgently.

Long non-coding RNAs (lncRNAs) are a class of RNAs consisted of 200 nucleotides. Generally, dysregulation of lncRNAs was implicated in tumorigenesis $(4,5)$. Long stress-induced non-coding transcript 5 (LSINCT5) plays a crucial role in regulating cell proliferation (6). Previous studies indicated LSINCT5 was involved in various cancers, including osteosarcoma (7), gastric cancer $(8,9)$, bladder cancer (10) and ovarian cancer (11). It has been reported that patients with colorectal cancer or gastric cancer have a higher level of LSINCT5, and their survival rate is poor (12). Another study by Mansoori et al. suggested LSINCT5 was downregulated in the overweight-obese subgroup, which was considered mass associated with a reduction in BC risk $(13,14)$.

MicroRNAs (miRNAs), non-coding RNAs consisted of 20-22 nucleotides, play crucial roles in various cancers $(15,16)$. The roles of miRNAs are mainly realized by recognizing the 3 ' untranslated region (UTR) sequence of target genes, and the subsequent degradation of these targeted mRNAs or inhibition of their translation (17). miR-30a was reported to be involved in cell proliferation, apoptosis, motility in tumor cells (18-20). LncRNAs regulate gene expression by sponging miRNAs at the post transcriptional level during tumor progression (21). Nevertheless, whether LSINCT5 combined with miR-30a to co-regulate $\mathrm{BC}$ remains unknown.

In this study, the aim was designed to investigate the role of LSINCT5 in BC and its regulatory molecular mechanism. The present study indicated a novel regulatory mechanism that LSINCT5 inhibited proliferation and motility of MCF-7 cells by inactivation of the Wnt/ $\beta$-catenin pathway sponging miR-30a in vitro and in vivo. The study proved that LSINCT5 might be a promising target for BC therapy. We present the following article in accordance with the ARRIVE reporting checklist (available at http://dx.doi.org/10.21037/atm-20-7253).

\section{Methods}

\section{Patient samples}

The subjects were 53 patients (clinical and follow-up data) with BC who underwent tumor resection in The Eighth Affiliated Hospital Sun Yat-sen University from September 2012 to February 2015. Prior to obtaining these fresh tissue specimens, all patients signed a written informed consent form. All BC tumors were confirmed by two pathologists, and $\mathrm{BC}$ grade and stage were classified according to the International Union Against Cancer guidelines (22). BC specimens and corresponding non-cancerous tissues were collected and stored at $-80^{\circ} \mathrm{C}$. All procedures performed in this study involving human participants were in accordance with the Declaration of Helsinki (as revised in 2013). This study was approved by the ethics committee of Sun Yat-sen University.

\section{Cell culture}

Human BC cell lines (MCF-7, MDA-MB-231, MDAMB-468, and SKBR-3) and normal breast epithelial cell line MCF-10A were obtained from the Type Culture Collection of the Chinese Academy of Science (CCTCC, Wuhan, China), and cultivated in Dulbecco's modified Eagle's medium or RPMI-1640 medium (Gibco, Grand Island, NY, USA) supplemented with $10 \%$ fetal bovine serum (FBS), $100 \mathrm{U} / \mathrm{mL}$ penicillin, and $100 \mu \mathrm{g} / \mathrm{mL}$ streptomycin under $\mathrm{pH}$ 7.2. (Invitrogen, Grand Island, NY, USA). Cells were cultured at $37^{\circ} \mathrm{C}$ with $5 \% \mathrm{CO}_{2}$ in a humid incubator.

\section{Cell transfection}

The short hairpin RNA (shRNA) specifically targeting LSINCT5 (called sh-LSINCT5) was synthesized by GenePharma (Shanghai, China), and nonspecific shRNA was used as negative control (sh-NC). The full-length sequence of LSINCT5 was inserted into pcDNA3.1 vector (pcDNA-LSINCT5, Invitrogen, Grand Island, NY, USA) and an empty pcDNA vector as negative control. The miR-30a mimic, miR-30a inhibitor, and corresponding NC were synthesized by RiboBio (Guangzhou, China). According to the manufacturer's protocol, MCF-7 cells 
Table 1 The primer sequence of genes

\begin{tabular}{ll}
\hline Gene & Sequence (5' to 3') \\
\hline LSINCT5 & F: CTCTAGGACTAACGCCTAGGCA \\
& R: TCGCCATCGGACAATCGGCTCGG \\
GAPDH & F: GTCAGGATCCACTCATCACG \\
& R: GATCGGACTTACGGACTCACATC \\
miR-30a & F: TCAGGGCTCAACGTACGGCGGC \\
& R: CCGTACCTAAGGTCGCTGCCTG \\
U6 & F: CTCGCTTCGGCAGCACA \\
& R: AACGCTTCACGAATTGCGT
\end{tabular}

was stably transfected with all plasmids for 48 hours using Lipofectamine 3000 reagent (Invitrogen, Grand Island, NY, USA).

\section{Quantitative real-time reverse transcription PCR (qRT- PCR)}

Total RNA was extracted from MCF-7 cells and tumor tissues with TRIzol reagent (Tiangen; Beijing, China) and monitored by a Real-Time PCR System (Applied Biosystems, USA) with FastKing One-Step RT-qPCR Kit according to the manufacturer's protocol (Tiangen; Beijing, China). PCR system according to the following procedure: $50{ }^{\circ} \mathrm{C}$ for 45 minutes for reverse transcription and $85{ }^{\circ} \mathrm{C}$ for 5 minutes for pre-degeneration. $94^{\circ} \mathrm{C}$ for 15 seconds, followed by 35 cycles at $60{ }^{\circ} \mathrm{C}$ for 40 seconds. Quantitative results were calculated with $2^{-\Delta \Delta C \mathrm{Ct}}$. DNA amplification was repeated three times. The primers were listed in Table 1. GAPDH or U6 was used as an internal reference.

\section{Plate clone formation assay}

MCF-7 cells were seeded in a 6-well plate at a density of 250 cells/well and incubated at $37^{\circ} \mathrm{C}, 5 \% \mathrm{CO}_{2}$ for ten days. After fixed with methanol and stained with crystal violet solution for 30 minutes, cells were cleaned with water and air-dried. The clones with $>150$ cells were counted with an optical microscope, and the clone formation rate was calculated as earlier (23). All results were repeated at least three times.

\section{Dual-luciferase reporter assay}

The 3' UTR segments of LSINCT5 (wt and mut) containing the predicted binding site for miR-30a were amplified and inserted into the pmirGLO- vector ( Promega, Madison, WI, USA). The primary sequence of LSINCT5 was listed as below: forward, 5'-CTCGAGTG TAGTCCCAGCTACTCAG-3'; reverse, 5'-GACGCGT TGGAGACCATCCTGGCTAACA-3'. MCF-7 cells were co-transfected with LSINCT5 (wt or mut) and miR-30a mimics or NC, respectively, followed by lysis for analyzing the relative luciferase activity $48 \mathrm{~h}$ later, according to the manufacturer's protocol (Promega, Madison, WI, USA). All results were repeated at least three times.

\section{Western blot assay}

Cells and tumor tissues were lysed with RIPA buffer (sc24948, Santa Cruz Biotechnology, USA). After separation by $12 \%$ SDS polyacrylamide gel, the corresponding proteins were moved to polyvinylidene difluoride (PVDF) membranes (Millipore, IPFL00010, Billerica, MA, USA). Primary antibodies specific to Ki-67 (sc-23900, 1:1,000, Santa Cruz Biotechnology, USA), caspase-3 (sc-271759, 1:1,000, SCB, USA), E-cadherin (sc-8426, 1:1,000, SCB, USA), Vimentin (sc-80975, 1:1,000, SCB, USA), $\mathrm{N}$-cadherin (sc-8424, 1:1,000, SCB, USA), $\beta$-catenin (\#2698, 1:1,000, Cell Signaling Technology, USA), TCF4 (\#2953, 1:1,000, CST, USA), c-Myc (\#9402, 1:1,000, CST, USA), GAPDH (sc-66163, 1:1,000, SCB, USA) were applied. After that, matched secondary antibodies incubated membranes samples (sc-516102, SCB, USA) and examined with enhanced chemiluminescence (ECL) substrate kit (Amersham Biosciences). Image J software analyzed the protein levels. All results were repeated at least three times.

\section{Transwell assay}

Transwell assay was employed to investigate invasion ability. After transfection with sh-LSINCT5 or miR30a inhibitor and in combination, MCF-7 cells were incubated in serum-free medium for $24 \mathrm{~h}$. Then, the cell suspension was collected and seeded into upper chambers (200 mL per chamber). Meanwhile, the lower chambers were supplemented with $600 \mu \mathrm{L}$ medium holding $10 \%$ FBS. Post incubation at $37^{\circ} \mathrm{C}$ for $48 \mathrm{~h}$, the chambers were pulled out and washed with PBS twice. A cotton swab was used to expunge the residual cells on the upper membranes. After fixed with $95 \%$ alcohol and stained with $0.1 \%$ crystal violet, cells were observed under an optical microscope (Leica, Germany), and the average invasive cell number was recorded. All results were repeated three times. 

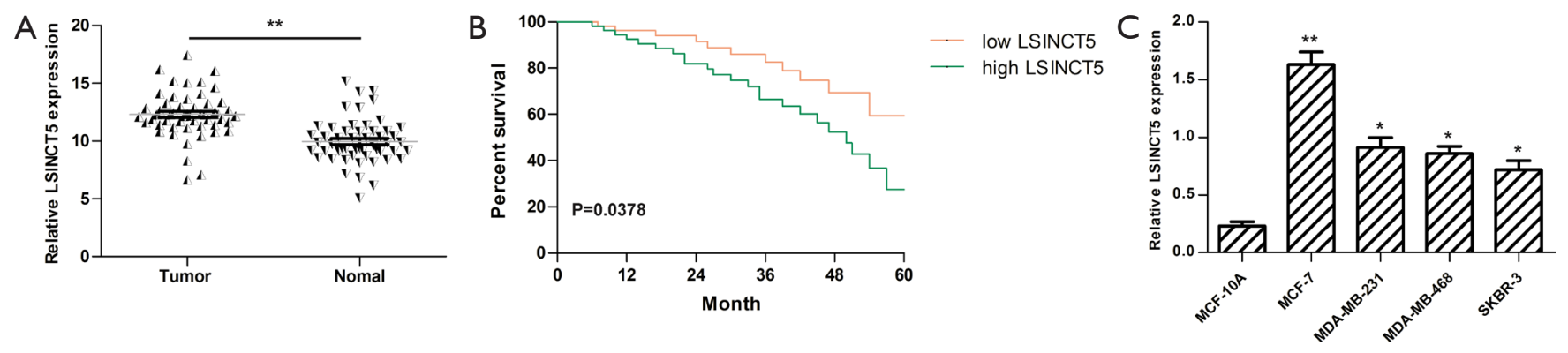

Figure 1 LSINCT5 is highly expressed in BC. (A) LSINCT5 level in BC tissues and corresponding non-cancerous tissues. (B) Survival rate in BC patients based on LSINCT5 level. (C) LSINCT5 level in BC cell lines and normal breast epithelial cell line. ${ }^{*}, \mathrm{P}<0.05,{ }^{* *}, \mathrm{P}<0.01 v s$. matched group. BC, breast cancer.

\section{Animal model}

In vivo model experiments were performed following the NIH Guide for the Care and Use of Laboratory Animals (National Academy of Sciences Press, 2011) and were approved by the ethics committee of Sun Yat-sen University. A total of $30 \mathrm{BALB} / \mathrm{c}$ nude mice (4-6 weeks old, 16-22 g) were obtained from the Animal Center of The Second Hospital of Lanzhou University and housed in a controlled environment at $18-22{ }^{\circ} \mathrm{C}$, humidity $55-60 \%$, in a 12-h light/dark cycle with free access to get food and water. Untreated MCF-7 cells or MCF-7 cells transfected with sh-LSINCT5 or sh-NC were subcutaneously injected into the ventral regions of mice to form tumors. Post successful modeling, mice were divided into three groups $(\mathrm{n}=10)$ : control group, sh-NC group, sh-LSINCT5 group. Then, the tumor weight and size [volume $=($ maximum diameter $\times$ minimum diameter $\left.{ }^{2}\right) / 2$ ] were evaluated after 30 days post-injection. Thirty days after injection, mice were euthanized with pentobarbital sodium $(200 \mathrm{mg} / \mathrm{kg}$ body weight) by intraperitoneal injection. Tumors were collected for further study.

\section{Immunobistochemistry (IHC)}

IHC assay was conducted to investigate the expression of Ki-67 and Vimentin in tumor-bearing mice. After dewaxing and rehydration, $4 \mu \mathrm{m}$ sections were incubated with primary antibodies (Ki-67, \#9449, 1:500, CST, USA; Vimentin, \#5741, 1:200, CST, USA) overnight at $4{ }^{\circ} \mathrm{C}$. The next day, corresponding secondary antibodies were added according to the manufacturer's protocol. The visual images were observed under a light microscope.

\section{Statistical analysis}

The statistical analysis was conducted with SPSS 21.0 (IBM, Armonk, USA). Measurement data were shown as mean $\pm \mathrm{SD}(\bar{x} \pm s)$, and the student's $t$-test analyzed data, and it is consistent with the normal distribution. The chisquare test was expressed by $\chi^{2}$, and Kaplan-Meier (K-M) analysis survival curve was used to draw the total survival. Data analysis of multiple sets was undertaken with Oneway ANOVA followed by Bonferroni post-hoc test. Enumeration data were provided in a percentage or ratio and verified by Chi-Square test. $\mathrm{P}<0.05$ showed statistical significance.

\section{Results}

\section{LSINCT5 was highly expressed in BC and positively correlated with the progression of $B C$}

As shown in Figure 1A, the level of LSINCT5 in tumor tissues was obviously higher than that in adjacent normal ones. Additionally, K-M analysis revealed that the high expression of LSINCT5 indicated an unfavorable prognosis of patients with breast cancer (Figure 1B). We found a clear correlation of LSINCT5 level with tumor stage and lymph node metastasis, and distant metastasis in patients with BC, but not with age and tumor size in those patients (Table 2). Also, the level of LSINCT5 in BC cell lines (MCF-7, MDA-MB-231, MDA-MB-468, and SKBR-3) and normal breast epithelial cell line MCF-10A was detected by RTqPCR. Results in Figure 1C showed that the expressions of LSINCT5 in BC cell lines was up-regulated, which was consistent with those in tissues. Notably, the differential 
Table 2 Relationship of LSINCT5 expression with clinicopathologic characteristics of $\mathrm{BC}$

\begin{tabular}{|c|c|c|c|c|}
\hline \multirow{2}{*}{ Variables } & \multirow{2}{*}{$\begin{array}{l}\text { Case } \\
(n=53)\end{array}$} & \multicolumn{2}{|c|}{ LSINCT5 expression } & \multirow{2}{*}{$P$ value } \\
\hline & & High $(n=27)$ & Low $(n=26)$ & \\
\hline \multicolumn{2}{|l|}{ Age (years) } & & & 0.398 \\
\hline$<60$ & 20 & 12 & 8 & \\
\hline$\geq 60$ & 33 & 15 & 18 & \\
\hline \multicolumn{2}{|c|}{ Tumor size (cm) } & & & 0.387 \\
\hline$<4$ & 18 & 11 & 7 & \\
\hline$\geq 4$ & 35 & 16 & 19 & \\
\hline \multicolumn{2}{|l|}{ T stage } & & & 0.012 \\
\hline $\mathrm{T} 1-\mathrm{T} 2$ & 21 & 6 & 15 & \\
\hline T3-T4 & 32 & 21 & 11 & \\
\hline \multicolumn{2}{|c|}{ Lymph node metastasis } & & & 0.018 \\
\hline No & 36 & 14 & 22 & \\
\hline Yes & 17 & 13 & 4 & \\
\hline \multicolumn{2}{|c|}{ Distant metastasis } & & & 0.028 \\
\hline No & 28 & 10 & 18 & \\
\hline Yes & 25 & 17 & 8 & \\
\hline
\end{tabular}

expression of LSINCT5 in MCF-7 cells was most significant. Therefore, we chose MCF-7 cells as further research object.

\section{LSINCT5 is a molecular sponge for miR-30ain BC cells}

To further assess the potential molecular mechanisms underlying LSINCT5 in BC, the StarBase v2.0 (http:// starbase.sysu.edu.cn/) and miRcode (http://mircode.org/) online tools were used to predict the potential binding partners. miR-30a was predicted to be a potential target for LSINCT5 (Figure 2A). QRT-PCR checked MiR-30a. As shown in Figure 1B, compared with negative control (shNC), LSINCT5 was successfully knocked down in MCF7 cells transfected with specific shRNA (sh-LSINCT5). Besides, knockdown LSINCT5 by sh-RNA noticeably upregulated miR-30a level, while overexpression of LSINCT5 had opposite effects. In the sh-LSINCT5 group. A reversed result was observed in the miR-30a inhibitor group. (Figure 2B,C,D) Consequently, the luciferase report assay was used to confirm the relationship between LSINCT5 and miR-30a further. There was a significant down-expression in LSINCT5 wt and miR-30a mimic group, while there was no markedly effects were observed in LSINCT5 mut and miR-30a mimic group (Figure 2E). These results, in short, showed that miR-30a targeted LSINCT5 and negatively regulated LSINCT5 in MCF-7 cells.

\section{Knockdown LSINCT5 inbibited cell proliferation by regulating miR-30a in MCF-7 cells}

Plate clone formation and Western blot assays were employed to investigate the proliferation ability of LSINCT5 on MCF-7 cells. As shown in Figure 3 A, compared with the control group, the plate clone formation rate was observably decreased in the sh-LSINCT5 group, while the addition of miR-30a inhibitor elevated colonyforming rate estimate from $38 \%$ to $79 \%$ in MCF-7 cells after a 10-day culture. Besides, western blot assay exhibited the cells transfected with sh-LSINCT5 were markedly elevated in the $\mathrm{Ki}-67$ protein level and decreased the cleaved caspase-3 protein level in MCF-7 cells (Figure 3B). Collectively, all the results displayed that knockdown LSINCT5 by shRNA inhibited cell proliferation by regulating miR-30a in MCF-7 cells.

\section{Knockdown LSINCT5 suppressed cell motility by regulating miR-30a in MCF-7 cells}

Transwell and Western blot assays were employed to investigate cell motility. As shown in Figure 4A, Transwell assay displayed that knockdown LSINCT5 by sh-RNA markedly decreased invasive cells estimate from 52 to 12 compared to control, while miR-30a inhibitor counteracted the inhibition induced by sh-LSINCT5. Besides, cell morphology also changed significantly after knockdown LSINCT5 by sh-RNA. As shown in Figure 4B, in the control group, cells arranged loosely, and the morphology changed markedly. However, knockdown LSINCT5 by sh-RNA markedly alleviated the changes and increased cell adhesion and tightness. Furthermore, western blot exerted that transfection with sh-LSINCT5 markedly elevated E-cadherin (epithelial marker protein) level and decreased Vimentin and N-cadherin (mesenchymal marker proteins) levels in MCF-7 cells. However, transfection with miR-30a inhibitor had opposite effects (Figure 4C). The study altogether suggested that knockdown LSINCT5 suppressed cell motility by regulating miR-30a in MCF-7 cells.

B-catenin/TCF4/c-Myc pathway took part in the roles of 
A

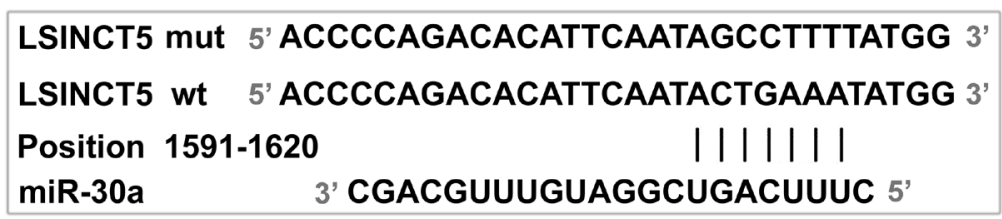

B

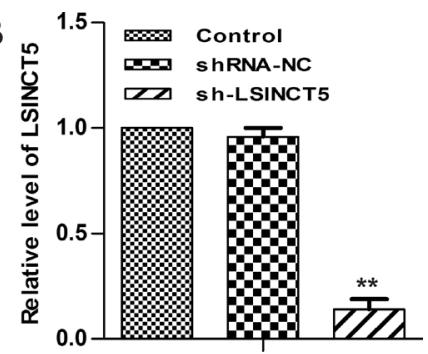

C

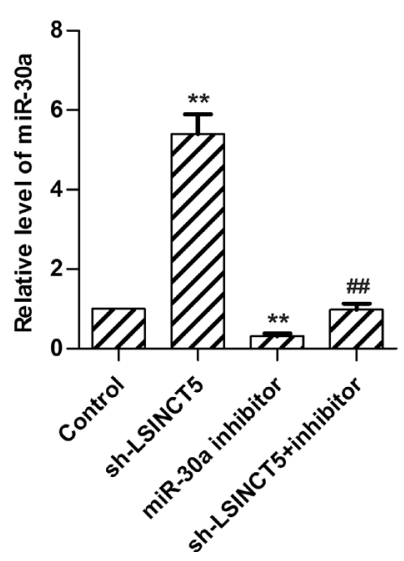

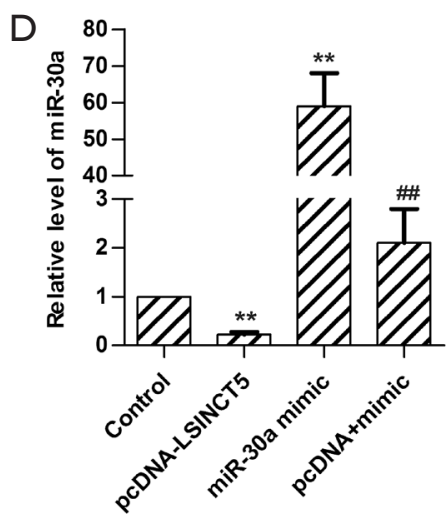

$\mathrm{E}$

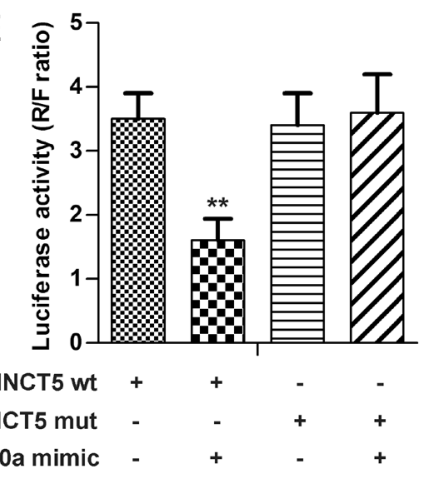

Figure 2 MiR-30a targeted LSINCT5 and negatively regulated LSINCT5 in MCF-7 cells. (A) The target site of miR-30a on LSINCT5 3'UTR (wt/mut). (B) LSINCT5 level in sh-LSINCT5 or NC transfected MCF-7 cells (**, $\mathrm{P}<0.01$ vs. control). (C) qRT-PCR monitored miR-30a. MiR-30a level in sh-LSINCT5 or miR-30a inhibitors and co-transfected MCF-7 cells ${ }^{* *}, \mathrm{P}<0.01$ vs. control, ${ }^{\#}, \mathrm{P}<0.01$ vs. miR30a inhibitor group). (D) miR-30a level in ApcDNA-LSINCT5 or miR-30a mimics and co-transfected MCF-7 cells $\left({ }^{* *}, \mathrm{P}<0.01\right.$ vs. control, \#, $\mathrm{P}<0.01$ vs. miR-30a mimic group). (E) Luciferase activity of LSINCT5 was monitored using dual-luciferase reporter in LSINCT5 (wt/ mut), and miR-30a mimic transfected MCF-7 cells. All experiments were conducted in triplicate. The images depicted a representative experiment, and the bar graph is a composite of three independent experiments ( ${ }^{* *}, \mathrm{P}<0.01 v s$. control). wt, wild type; mut, mutant type; qRT-PCR, quantitative real-time polymerase chain reaction; shRNA, short hairpin RNA; NC, negative control.

\section{LSINCTS}

A further mechanistic study was performed by using Western blot to investigate the underlying molecular mechanism of LSINCT5 on MCF-7 cells. $\beta$-catenin/ $\mathrm{TCF} 4 / \mathrm{c}-\mathrm{Myc}$ was a part of the Wnt signaling pathway. Therefore, the Wnt pathway antagonist (ICG-001) was utilized to confirm further the implication between LSINCT5 and $\beta$-catenin/TCF4/c-Myc pathway. As shown in Figure 5 A, overexpression of LSINCT5 significantly accelerated the expressions of $\beta$-catenin, TCF4, and c-Myc. With the addition of ICG-001, the protein levels of $\beta$-catenin, TCF4, and c-Myc were markedly decreased in MCF-7 cells. Besides, overexpression of LSINCT5 dramatically promoted cell proliferation (Figure $5 B$ ) and invasion (Figure 5C), while ICG-001 significantly inhibited the facilitation. Our study proved that $\beta$-catenin/TCF4/
c-Myc pathway participated in the roles of LSINCT5.

\section{Knockdown LSINCT5 restrained tumor growth and motility by inactivating $\beta$-catenin/TCF4/c-Myc patbway in vivo}

After feed 30 days, xenograft nude mice were sacrificed. The in-situ tumors were harvested and weighed. As shown in Figure $6 A, B$ the tumor weight and volume was significantly reduced in xenograft nude mice injected by MCF-7 cells with sh-LSINCT5, compared with the control group. Besides, qRT-PCR showed that after injection by MCF-7 cells with sh-LSINCT5, LSINCT5 level was reduced, while miR-30a was increased in xenograft nude mice (Figure 6C). The expression of $\mathrm{Wnt}$ pathway-related proteins ( $\beta$-catenin, TCF4, and c-Myc) was monitored by Western blot. As shown in Figure 


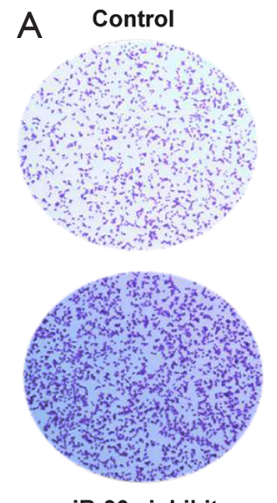

miR-30a inhibitor

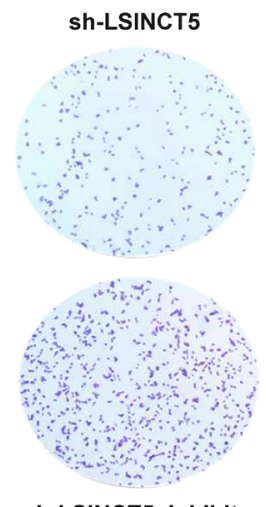

sh-LSINCT5+inhibitor
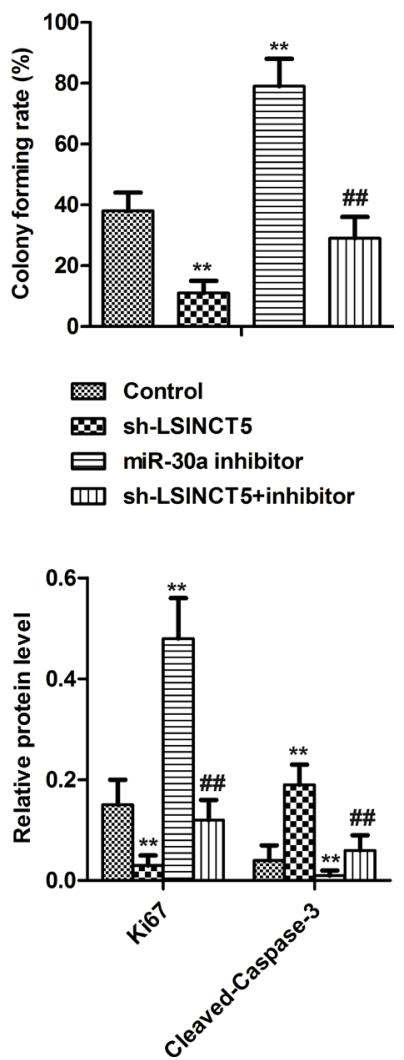

Figure 3 Knockdown LSINCT5 inhibited cell proliferation by regulating miR-30a in MCF-7 cells. MCF-7 cells were transfected with shLSINCT5 or miR-30a inhibitor and in combination. (A) Clone formation images of MCF-7 cells in each group, images were magnified at $20 \times$ (stained with $0.2 \%$ crystal violet solution). (B) The protein levels of $\mathrm{Ki}-67$ and cleaved-caspase- 3 were monitored in each group by Western blot. The GAPDH protein was employed as an internal reference. All experiments were conducted in triplicate. The images depicted a representative experiment, and the bar graph is a composite of three independent experiments. **, $\mathrm{P}<0.01$ vs. control, ${ }^{\# \#}, \mathrm{P}<0.01$ vs. miR-30a inhibitor group. sh-LSINCT5, short hairpin LSINCT5.

6D, knockdown LSINCT5 significantly inhibited the expressions of $\beta$-catenin, TCF4, and c-Myc. Furthermore, the results from the IHC assay exhibited that the expression of Ki-67 and Vimentin was reduced in tumors from shLSINCT5 group mice (Figure 6E). Together, we concluded knockdown LSINCT5 restrained tumor growth and motility by inactivating the $\beta$-catenin/TCF4/c-Myc pathway in vivo.

\section{Discussion}

As the most common tumor in women, BC caused approximately 410,712 deaths, and the patients who had BC have exceeded 4.4 million on a global scale (24). Encouragingly, the mortality has been reduced due to the application of tumor node metastasis (TNM) staging system and molecular diagnostic tests (25). However, the clinical application of these kits was still limited, owing to the high cost and regional availability (26). Therefore, it is the most intuitive way to investigate the potential molecular mechanism in the treatment of BC.

As critical regulators, lncRNAs play essential roles in cell proliferation, apoptosis, and metastasis in BC (27-29). Zhang et al. reported that overexpression of AFAP1-AS1 was implicated in poor survival, and knockdown AFAP1AS1 inhibited cell proliferation and motility, as well as induced cell apoptosis in BC (30). Besides, as reported by Basak et al., H19 played crucial roles in endocrine therapy resistance, and downregulating $\mathrm{H} 19$ contributed to alleviate Tamoxifen and Fulvestrant-resistance (31). Similarity, as a newly discovered lncRNA, LSINCT5 was also associated with cell proliferation in $\mathrm{BC}$ (12). Consistent with these 
A

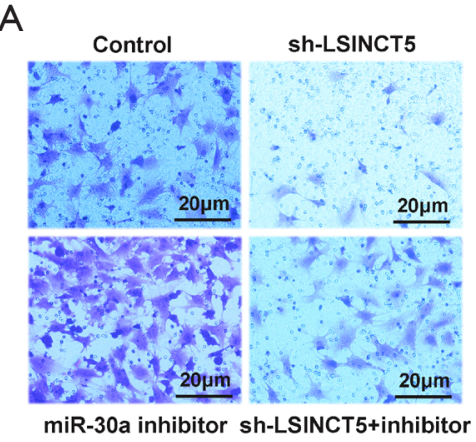

B

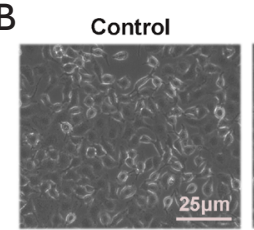

sh-LSINCT5
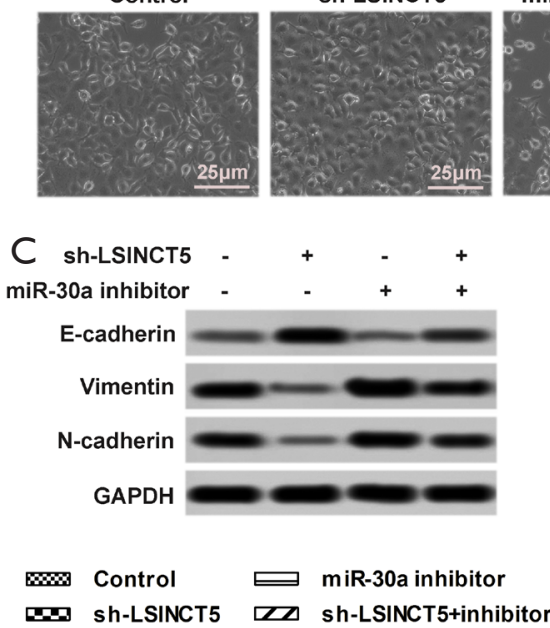

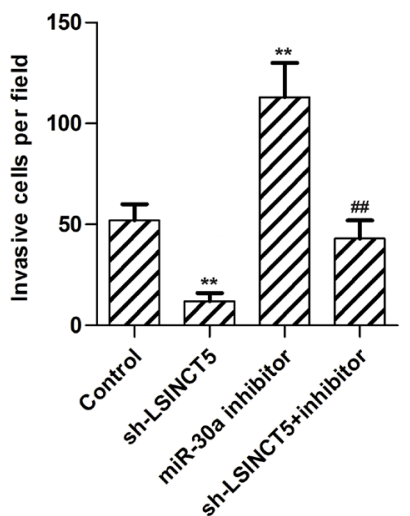

miR-30a inhibitor sh-LSINCT5+inhibitor
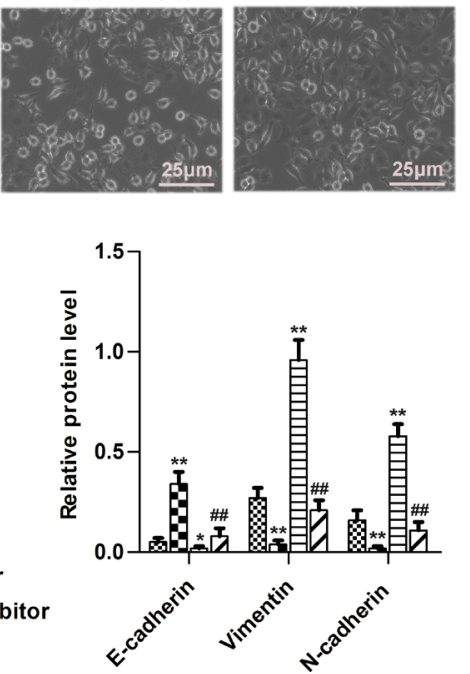

Figure 4 Knockdown LSINCT5 suppressed cell motility by regulating miR-30a in MCF-7 cells. MCF-7 cells were transfected with shLSINCT5 or miR-30a inhibitor and in combination. (A) Cell invasion images of MCF-7 cells in each group, images were magnified at 200x (stained with $0.1 \%$ crystal violet solution). (B) Microscopic images of MCF-7 cells in each group, images were magnified at 100×. (C) The protein levels of epithelial-mesenchymal transition (Vimentin, N-cadherin, and E-cadherin) were monitored in each group by Western blot. The GAPDH protein was employed as an internal reference. All experiments were conducted in triplicate. The images depicted a representative experiment, and the bar graph is a composite of three independent experiments. ${ }^{*}, \mathrm{P}<0.05,{ }^{* *}, \mathrm{P}<0.01 v s$. control, ${ }^{\# \prime}, \mathrm{P}<0.01 v s$. miR-30a inhibitor group. sh-LSINCT5, short hairpin LSINCT5.

results, in the present study, we found that LSINCT5 was highly expressed in BC tissues and cell lines, the 5-year survival of patients with high expression decreased. Further, knockdown LSINCT5 restrained cell proliferation, invasion, and EMT, which supported previous reports (6). Collectively, the present study proved that LSINCT5 played a carcinogenic role in $\mathrm{BC}$.

Nowadays, it is an excellent strategy to study the interaction between lncRNAs and miRNAs to investigate the mechanism of lncRNA-mediated tumorigenesis (32). Most studies have shown that lncRNA compete with
miRNA for original responses, causing changes in transcription and expression of miRNA. Low-expression of miR-30a was always observed in BC cells $(32,33)$. Zhang et al. reported that knockdown FEZF1-AS1 inhibited BC cell growth and motility by sponging miR-30a in vitro and vivo (34). Further study carried out by Kawaguchi et al. exhibited that overexpression of miR-30a was significantly implicated in better survival in both luminal and basallike BC, demonstrating miR-30a could improve survival of $\mathrm{BC}$ patient (35). Herein, our results are consistent with the previous study. In the present study, miR-30a was 

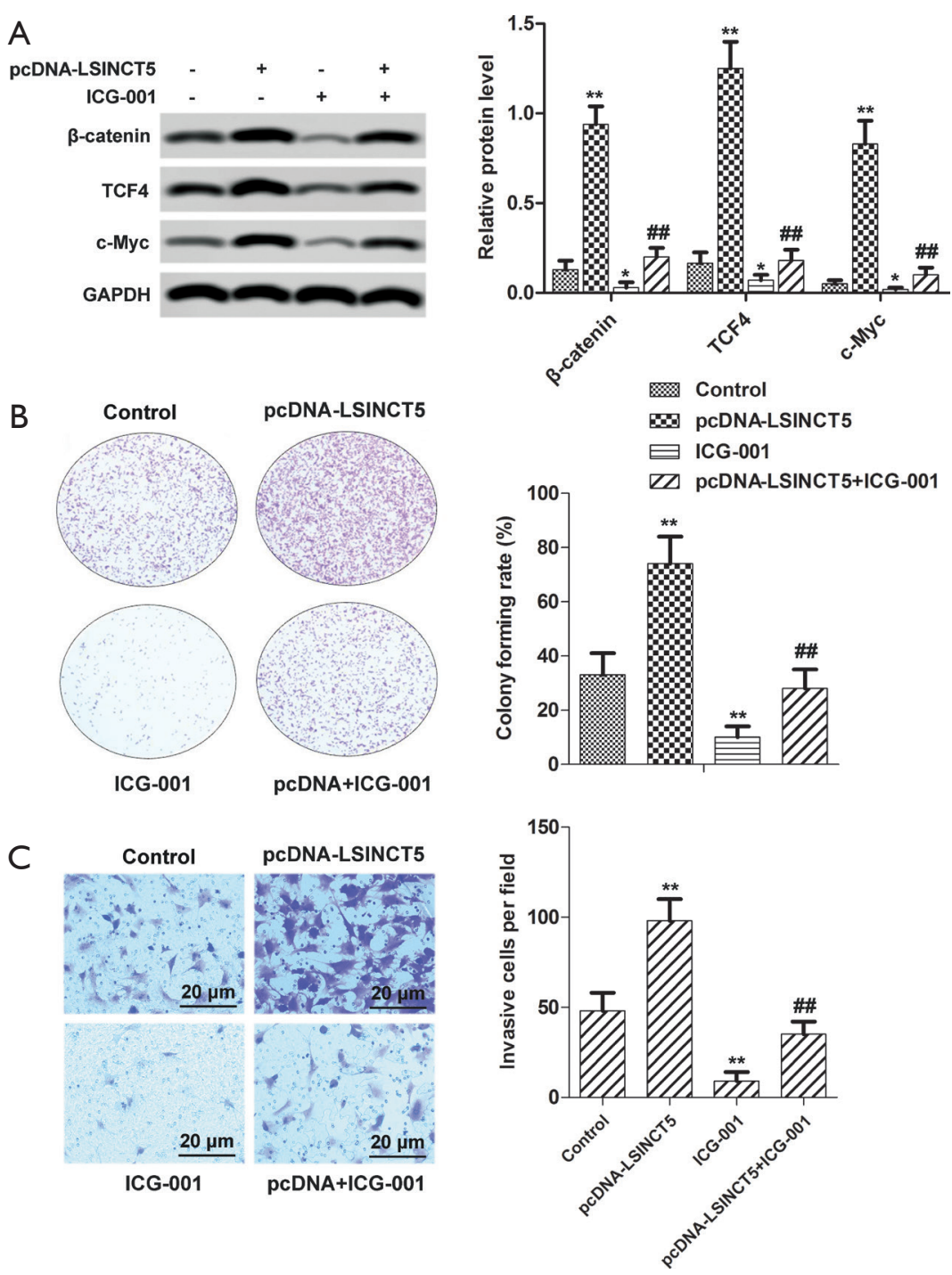

Figure $5 \beta$-catenin/TCF4/c-Myc pathway took part in the roles of LSINCT5. MCF-7 cells were transfected with pcDNA-LSINCT5 or ICG-001 and in combination with ICG-001. (A) The protein levels of Wnt pathway-related proteins ( $\beta$-catenin, TCF4, and c-Myc) were monitored in each group by Western blot. The GAPDH protein was employed as an internal reference. (B) Clone formation images of MCF-7 cells in each group, images were magnified at $20 \times$ (stained with $0.2 \%$ crystal violet solution). (C) Cell invasion images of MCF-7 cells in each group, images were magnified at $200 \times$ (stained with $0.1 \%$ crystal violet solution). All experiments were conducted in triplicate. The images depicted a representative experiment, and the bar graph is a composite of three independent experiments. ${ }^{*}, \mathrm{P}<0.05,{ }^{* *}, \mathrm{P}<0.01$ vs. control, ${ }^{\#}, \mathrm{P}<0.01$ vs. ICG-001 group. TCF4, transcription factor 4; c-Myc, cellular myelocytomatosis oncogene.

confirmed to be a target of LSINCT5, and the miR-30a level in BC cells after transfection of pcDNA-LSINCT5 or sh-LSINCT5 was also changed.. Additionally, the cell proliferation, invasion and EMT of miR-30a-inhibitor transfected MCF-7 cells were accelerated. Thus, these data indicated that LSINCT5 could regulate miR-30a to affect the growth and metastasis of BC cells.
Aberrant regulation of the $\mathrm{Wnt} / \beta$-catenin pathway accelerated tumor initiation and progression (36-40). Increasing evidence exhibited that various regulators, including $\beta$-catenin, TCF4, and c-Myc, participated in BC oncogenesis $(41,42)$. As a primary protein of Wnt signaling, $\beta$-catenin was phosphorylated by CK1 and GSK3 $\beta$ and recognized by $\beta$-TrCP. However, in the Wnt pathway 

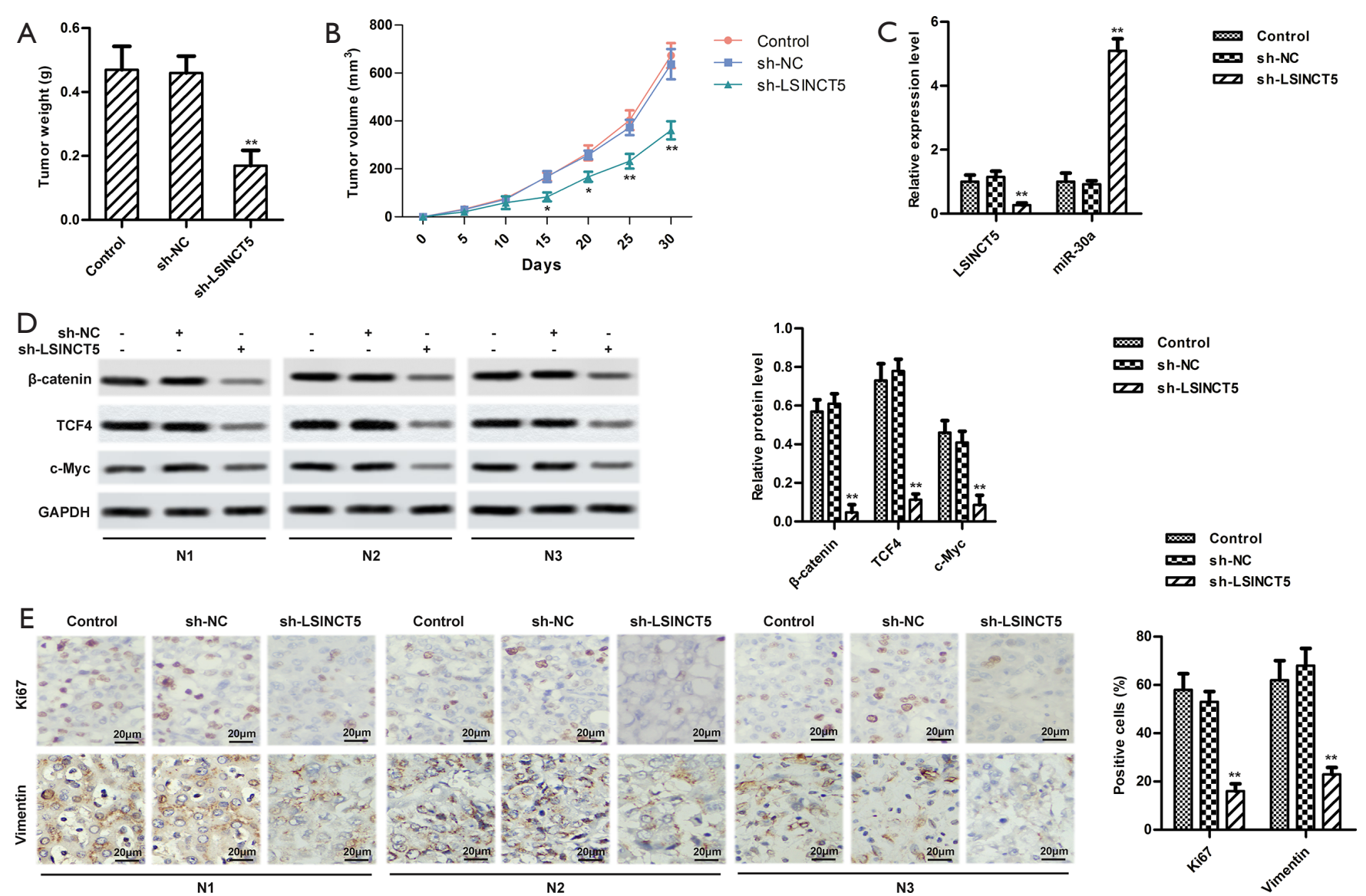

Figure 6 Knockdown LSINCT5 restrained tumor growth and motility by inactivating the $\beta$-catenin/TCF4/c-Myc pathway in vivo. MCF7 cells untreated or transfected with sh-LSINCT5 were injected into the ventral regions of mice to form tumors. Post successful modeling, mice were divided into three groups with 10 in each group: control group, sh-LSINCT5 group (mice were injected with MCF-7 cells transfected with sh-LSINCT5). (A) Tumor weight in 3 groups. (B) Tumor volume in three groups. (C) LSINCT5 and miR-30a were monitored by qRT-PCR. (D) The protein levels of Wnt pathway-related proteins ( $\beta$-catenin, TCF4, and c-Myc) were monitored in each group by Western blot. The GAPDH protein was employed as an internal reference. (E) Expression of Ki-67 and Vimentin in tumors were detected by IHC assay, images were magnified at 400x. * $\mathrm{P}<0.05,{ }^{* *}, \mathrm{P}<0.01$ vs. control. All experiments were conducted in triplicate. The images depicted a representative experiment, and the bar graph is a composite of ten independent experiments. sh-LSINCT5, short hairpin LSINCT5; sh-NC, short hairpin negative control; TCF4, transcription factor 4; c-Myc, cellular myelocytomatosis oncogene.

antagonist, $\beta$-catenin escaped from being phosphorylated and recognized, and the dephosphorylated $\beta$-catenin then translocated into the nucleus, worked together with TCF/LEF family to induce target gene expression (43). Therefore, in our study, we determined the expression levels of $\beta$-catenin, TCF4, and c-Myc. It was found that knockdown of LSINCT5 markedly inhibited the expression of $\beta$-catenin, and the enhanced $\beta$-catenin level further inactivated TCF4 and c-Myc expression. pcDNALSINCT5 cells significantly promoted $\mathrm{Wnt} / \beta$-catenin activation. All data presented that the roles of LSINCT5 in
$\mathrm{BC}$ were realized by regulating $\mathrm{Wnt} / \beta$-catenin pathway.

In conclusion, the present study showed that LSINCT5 regulated MCF-7 cell proliferation and motility by sponging miR-30a in vitro. The further mechanistic study indicated that roles of LSINCT5 in MCF-7 cells participated in the $\mathrm{Wnt} / \beta$-catenin pathway. The experiment in xenograft nude mice showed knockdown LSINCT5 targeted miR-30a to inhibit tumor growth and motility through modulating $\mathrm{Wnt} / \beta$-catenin pathway in vivo. The present results prove that designing targeted drugs against LSINCT5 supplies a new perspective for $\mathrm{BC}$ treatment. 


\section{Acknowledgments}

Funding: None.

\section{Footnote}

Reporting Checklist: The authors have completed the ARRIVE reporting checklist. Available at http://dx.doi. org/10.21037/atm-20-7253

Data Sharing Statement: Available at http://dx.doi. org/10.21037/atm-20-7253

Conflicts of Interest: Both authors have completed the ICMJE uniform disclosure form (available at http://dx.doi. org/10.21037/atm-20-7253). The authors have no conflicts of interest to declare.

Etbical Statement: The authors are accountable for all aspects of the work in ensuring that questions related to the accuracy or integrity of any part of the work are appropriately investigated and resolved. All procedures performed in this study involving human participants were in accordance with the Declaration of Helsinki (as revised in 2013). This study was approved by the ethics committee of Sun Yat-sen University and all patients signed a written informed consent form. Animal experiments were approved by the ethics committee of Sun Yat-sen University and were performed following the NIH Guide for the Care and Use of Laboratory Animals (National Academy of Sciences Press, 2011).

Open Access Statement: This is an Open Access article distributed in accordance with the Creative Commons Attribution-NonCommercial-NoDerivs 4.0 International License (CC BY-NC-ND 4.0), which permits the noncommercial replication and distribution of the article with the strict proviso that no changes or edits are made and the original work is properly cited (including links to both the formal publication through the relevant DOI and the license). See: https://creativecommons.org/licenses/by-nc-nd/4.0/.

\section{References}

1. Hong W, Dong E. The past, present and future of breast cancer research in China. Cancer Lett 2014;351:1-5.

2. Ricci F, Le Tourneau C. Emerging new predictive

biomarkers in metastatic breast cancer: Caveolin-1 and weekly nab-paclitaxel plus gemcitabine, are we on for tomorrow? Chin Clin Oncol 2019;8:S14.

3. Król M, Pawłowski KM, Majchrzak K, et al. Why chemotherapy can fail? Pol J Vet Sci 2010;13:399-406.

4. Chen J, Ao L, Yang J. Long non-coding RNAs in diseases related to inflammation and immunity. Ann Transl Med 2019;7:494.

5. Meng $\mathrm{YB}, \mathrm{He} \mathrm{X}$, Huang $\mathrm{YF}$, et al. Long non-coding RNA CRNDE promotes multiple myeloma cell growth by suppressing miR-451. Oncol Res 2017;25:1207-14.

6. Silva JM, Boczek NJ, Berres MW, et al. LSINCT5 is over expressed in breast and ovarian cancer and affects cellular proliferation. RNA Biol 2011;8:496-505.

7. Kong D, Li C, Yang Q, et al. Long noncoding RNA LSINCT5 acts as an oncogene via increasing EZH2induced inhibition of APC expression in osteosarcoma. Biochem Biophys Res Commun 2018;507:193-7.

8. Xu Mi-Die, Qi P, Weng WW, et al. Long non-coding RNA LSINCT5 predicts negative prognosis and exhibits oncogenic activity in gastric cancer. Medicine (Baltimore) 2014;93:e303.

9. Qi P, Lin WR, Zhang M, et al. E2F1 induces LSINCT5 transcriptional activity and promotes gastric cancer progression by affecting the epithelial-mesenchymal transition. Cancer Manag Res 2018;10:2563-71.

10. Zhu X, Li Y, Zhao S, et al. LSINCT5 activates Wnt/ $\beta$-catenin signaling by interacting with NCYM to promote bladder cancer progression. Biochem Biophys Res Commun 2018;502:299-306.

11. Long X, Li L, Zhou Q, et al. Long non-coding RNA LSINCT5 promotes ovarian cancer cell proliferation, migration and invasion by disrupting the CXCL12/ CXCR4 signalling axis. Oncol Lett 2018;15:7200-6.

12. Xu MD, Qi P, Weng WW, et al. Long non-coding RNA LSINCT5 predicts negative prognosis and exhibits oncogenic activity in gastric cancer. Medicine 2014;93:e303.

13. Mansoori Y, Tabei MB, Askari A, et al. Expression levels of breast cancer-related GAS5 and LSINCT5 lncRNAs in cancer-free breast tissue: Molecular associations with age at menarche and obesity. Breast J 2018;24:876-82.

14. Horn-Ross PL, Canchola AJ, Bernstein L, et al. Lifetime body size and estrogen-receptor-positive breast cancer risk in the California Teachers Study cohort. Breast Cancer Res 2016;18:132.

15. Luo Z, Li D, Luo X, et al. Decreased expression of miR$548 c-3 p$ in osteosarcoma contributes to cell proliferation 
via targeting ITGAV. Cancer Biother Radiopharm 2016;31:153-8.

16. Zhang X, Li M, Zuo K, et al. Upregulated miR-155 in papillary thyroid carcinoma promotes tumor growth by targeting APC and activating Wnt/beta-catenin signaling. J Clin Endocrinol Metab 2013;98:E1305-13.

17. Croce CM, Calin GA. miRNAs, Cancer, and Stem Cell Division. Cell 2005;122:6-7.

18. Pan Y, Tong S, Cui R, et al. Long non-coding MALAT1 functions as a competing endogenous RNA to regulate Vimentin expression by sponging miR-30a$5 \mathrm{p}$ in hepatocellular carcinoma. Cell Physiol Biochem 2018;50:108-20.

19. Luan N, Wang Y, Liu X. Absent expression of miR-30a promotes the growth of lung cancer cells by targeting MEF2D. Oncol Lett 2018;16:1173-9.

20. Correction: Heterochromatin Protein HP1 Promotes Colorectal Cancer Progression and Is Regulated by miR30a. Cancer Res 2018;78:3741.

21. Yang $\mathrm{C}, \mathrm{Wu} \mathrm{D}, \mathrm{Gao} \mathrm{L}$, et al. Competing endogenous RNA networks in human cancer: hypothesis, validation, and perspectives. Oncotarget 2016; 7: 13479-90..

22. Sobin LH, Hermanek P, Hutter RV. TNM classification of malignant tumors. A comparison between the new (1987) and the old editions. Cancer 1988;61:2310-4.

23. Li T, Zhang C, Ding Y, et al. Umbilical cord-derived mesenchymal stem cells promote proliferation and migration in MCF-7 and MDA-MB-231 breast cancer cells through activation of the ERK pathway. Oncol Rep 2015;34:1469-77.

24. Veronesi U, Boyle P, Goldhirsch A, et al. Breast cancer. Lancet 2005;365:1727-41.

25. Kraeima J, Siesling S, Vliegen IMH, et al. Individual risk profiling for breast cancer recurrence: towards tailored follow-up schemes. Br J Cancer 2013;109:866-71.

26. Drukker CA, Bueno-de-Mesquita JM, Retel VP, et al. A prospective evaluation of a breast cancer prognosis signature in the observational RASTER study. Int J Cancer 2013;133:929-36.

27. Lv P, Qiu X, Gu Y, et al. Long non-coding RNA SNHG6 enhances cell proliferation, migration and invasion by regulating miR-26a-5p/MAPK6 in breast cancer. Biomed Pharmacother 2019;110:294-301.

28. Gao H, Hao G, Sun Y, et al. Long noncoding RNA H19 mediated the chemosensitivity of breast cancer cells via Wnt pathway and EMT process. Onco Targets Ther 2018;11:8001-12.

29. Li RH, Chen M, Liu J, et al. Long noncoding RNA
ATB promotes the epithelial-mesenchymal transition by upregulating the miR-200c/Twist1 axe and predicts poor prognosis in breast cancer. Cell Death Dis 2018;9:1171.

30. Zhang K, Liu P, Tang L, et al. AFAP1-AS1 promotes epithelial-mesenchymal transition and tumorigenesis through Wnt/B-Catenin signaling pathway in triplenegative breast cancer. Front Pharmacol 2018;9:1248.

31. Basak P, Chatterjee S, Bhat V, et al. Long Non-Coding RNA H19 Acts as an Estrogen Receptor Modulator that is Required for Endocrine Therapy Resistance in ER+ Breast Cancer Cells. Cell Physiol Biochem 2018;51:1518-32.

32. Kawaguchi T, Yan L, Qi QY, et al. Overexpression of suppressive microRNAs, miR-30a and miR-200c are associated with improved survival of breast cancer patients. Sci Rep 2017;7:15945.

33. Zhang HD, Jiang LH, Sun DW, et al. miR-30a inhibits the biological function of breast cancer cells by targeting Notch1. Int J Mol Med 2017;40:1235-42.

34. Zhang Z, Sun L, Zhang Y, et al. Long non-coding RNA FEZF1-AS1 promotes breast cancer stemness and tumorigenesis via targeting miR-30a/Nanog axis. J Cell Physiol 2018;233:8630-8.

35. Kawaguchi T, Yan L, Qi Q, et al. Overexpression of suppressive microRNAs, miR-30a and miR-200c are associated with improved survival of breast cancer patients. Sci Rep 2017;7:15945.

36. Wang J, Qi H, Zhang X, et al. Saikosaponin D from Radix Bupleuri suppresses triple-negative breast cancer cell growth by targeting $\beta$-catenin signaling. Biomed Pharmacother 2018;108:724-33.

37. Fang Y, Yuan Y, Zhang LL, et al. Downregulated GBX2 gene suppresses proliferation, invasion and angiogenesis of breast cancer cells through inhibiting the $\mathrm{Wnt} / \beta$-catenin signaling pathway. Cancer Biomark 2018;23:405-18.

38. Shi S, Chen X, Liu H, et al. LGR5 acts as a target of miR$340-5 \mathrm{p}$ in the suppression of cell progression and drug resistance in breast cancer via $\mathrm{Wnt} / \beta$-catenin pathway. Gene 2019;683:47-53.

39. Yang F, Xiao Z, Zhang S. Knockdown of miR-194-5p inhibits cell proliferation, migration and invasion in breast cancer by regulating the $\mathrm{Wnt} / \beta$-catenin signaling pathway. Int J Mol Med 2018;42:3355-63.

40. Jiang Q, He M, Guan S, et al. MicroRNA-100 suppresses the migration and invasion of breast cancer cells by targeting FZD-8 and inhibiting Wnt/ $\beta$-catenin signaling pathway. Tumour Biol 2016;37:5001-11.

41. Zeng B, Li Y, Feng Y, et al. Downregulated miR-1247$5 \mathrm{p}$ associates with poor prognosis and facilitates tumor 
cell growth via DVL1/Wnt/ $\beta$-catenin signaling in breast cancer. Biochem Biophys Res Commun 2018;505:302-8.

42. Wang J, Qi H, Zhang X, et al. Saikosaponin D from Radix Bupleuri suppresses triple-negative breast cancer cell growth by targeting beta-catenin signaling. Biomed Pharmacother 2018;108:724-33.

Cite this article as: Zhang $G$, Song $W$. Long non-coding RNA LSINCT5 inactivates $W n t / \beta$-catenin pathway to regulate MCF-7 cell proliferation and motility through targeting the miR-30a. Ann Transl Med 2020;8(24):1635. doi: 10.21037/atm20-7253
43. Mir R, Pradhan SJ, Patil P, et al. Wnt/ $\beta$-catenin signaling regulated SATB1 promotes colorectal cancer tumorigenesis and progression. Oncogene 2016;35:1679-91.

(English Language Editor: J. Chapnick) 